\title{
High level measurement comparison on a high performance multifunction calibrator between THE INRIM-Lab for multifunction instruments calibration and an Accredited Electrical Calibration Laboratory
}

\author{
F. Galliana ${ }^{1}$, M. Lanzillotti' and G. La Paglia ${ }^{3}$ \\ 1, 2,3 National Institute of Metrological Research, strada delle Cacce 91, 10135 TURIN - Italy \\ 1+390113919336, f.galliana@inrim.it \\ 2+390113919315, m.lanzillotti@inrim.it \\ 33390113919420, g.lapaglia@inrim.it
}

\begin{abstract}
Résumé. Cet article présente une comparaison de mesures entre le laboratoire pour l'étalonnage des instruments électriques multifonctions de l'Institut national de métrologie de recherche (INRIM) et un laboratoire d'étalonnage électrique de haut niveau. L'instrument à étalonner utilisé est un calibrateur multifonction de haute performance qui a été choisi pour l'amplitude de ses champs de mesure et son meilleur définissabilité (incertitude intrinsèque) que celle d'un multimètre numérique. L'analyse des résultats a été effectuée en évaluant le degré de corrélation entre les mesures des deux laboratoires. L'erreur-type était inférieur à 1 pour tous les points de mesure.
\end{abstract}

\section{Introduction}

High accuracy and high stability multifunction instruments as digital multimeters (DMMs) and multifunction calibrators (MFCs) play an important role to grant the metrological capabilities of low frequency Electrical Calibration Laboratories [1]. They operate in the five main low frequency electrical quantities, (dc and ac voltage, dc and ac current and dc resistance) in wide measurement fields and with high accuracy and can be calibrated by means of alternative methods, the easiest of which is the "artifact calibration", that requires only few Reference Standards [2-4]. Another method that allows the adjustment in a higher number of measurement points is described in $[5,6]$. Nowadays, electrical calibration Laboratories have also the need to calibrate and adjust high performance DMMs and MFCs for their customers. For this reason, these Laboratories are now equipped with the best commercially available instrumentation and develop calibration processes to significantly reduce their uncertainty levels. To assure the reliability of their measurement results these Laboratories have to be accredited by an Accreditadion Body. On the other hand, the Accreditadion Bodies need to verify these Laboratories according to the requested accuracy level. To accomplish to this task, the main check is made by means of interlaboratory comparisons (ILC's). The challenge is to have instruments, expertise and means to perform significant ILCs to correctly verify the capabilities of these Laboratories. ILCs between National Metrology Institues (NMIs) and accredited Laboratories using as travelling Standards fixed electrical Standards as in [7-9] unfortunately don't cover the wideness of the measurement operating fields of these Laboratories. A traveling standard covering wide fields and used by INRIM since several years for ILCs with accredited electrical calibration Laboratories is the $8^{1 / 2}$ digit DMM $[1,10,11]$. With this instrument it is possible to correctly verify the capabilities of medium-high level calibration Laboratories. These Laboratories are equipped of high level multifunction instruments as Reference Standards to be calibrated at NMIs as in the traceability chart of fig. 1 .
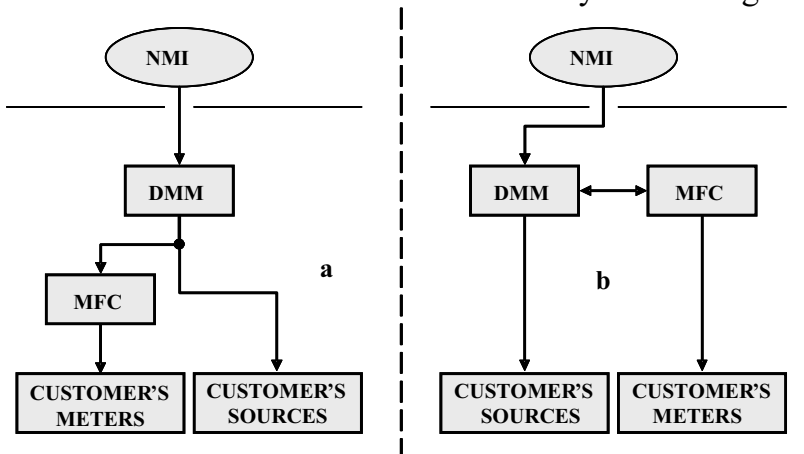

Figure 1 Traceability schemes from a NMI to electrical Laboratories through a high precision DMM. In a) the DMM acts as primary Reference standard, while in b) the DMM acts as transfer standard [1].

Unfortunately, ILCs with a $8^{1 / 2}$ digit DMM don't allow to correctly verify high level calibration Laboratories. These Laboratories are equipped of complete sets of Reference Standards to be calibrated at at NMIs as in the traceability chart of fig. 2.

f.galliana@inrim.it 


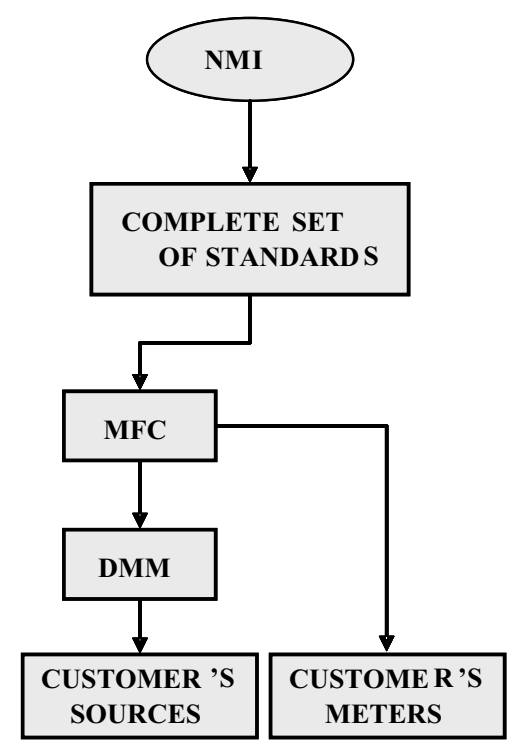

Figure 2. Traceability scheme from a NMI to an electrical Laboratory trough a complete set of primary standards.

These Standards are for example a $10 \mathrm{~V} \mathrm{Dc}$ Voltage Reference standard, voltage dividers, standard resistors and shunts, and Ac/Dc Voltage Transfer Standard), with which to perform the adjustment and calibration of their MFC(s) and DMM(s). Nevertheless, till now, these high level Laboratories have been verified by means of ILCs on both a $8^{1 / 2}$ digit DMM and some fixed Standards as a $10 \mathrm{~V}$ or a $10 \mathrm{k} \Omega$ Reference Standards. Nevertheless, this kind of ILCs is not enough significant in terms of uncertainty levels and wideness of the measurement fields to verify correctly these high level Laboratories. In this paper, an ILC between INRIM and a high level electrical calibration Laboratory under evaluation for its accreditation renewal on a high level MFC is presented. This instrument was chosen for its wide measurement fields and its better definability and definitional uncertainty [12] than a $8^{1 / 2}$ digit DMM.

\section{The travelling Standard of the ILC}

In the described ILC a high performance MFC J. Fluke 5700 A (Fig. 3) with associate the transconductance amplifier J. Fluke 5725 A was involved as travelling Standard to be calibrated by the INRIM-Lab for multifunction instruments calibration and by a high level accredited Electrical Calibration Laboratory.

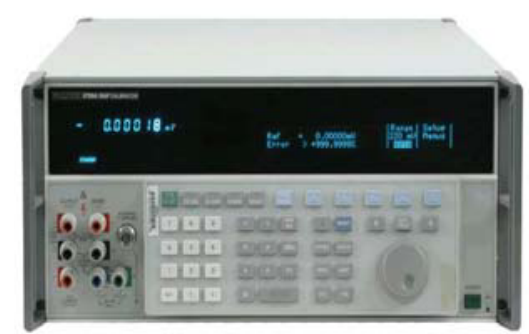

Figure 3. Multifunction calibrator J. Fluke mod. 5700A under calibration in the comparison.
The high performance 5700A Calibrator covers a wide portion of the calibration requests of electrical calibration Laboratories. With it, it is possible to calibrate a wide variety of DMMs. It operates in DC Voltage up to $1100 \mathrm{~V}$ and in $\mathrm{AC}$ Voltage from $1 \mathrm{mV}$ to $1100 \mathrm{~V}$ at frequencies from $10 \mathrm{~Hz}$ to $1.2 \mathrm{MHz}$. In the resistances function it operates from $1 \Omega$ to $100 \mathrm{M} \Omega$. In DC and $\mathrm{AC}$ current it works from $1 \mu \mathrm{A}$ to $10 \mathrm{~A}$, and at frequencies for $\mathrm{AC}$ current from $10 \mathrm{~Hz}$ to $10 \mathrm{kHz}$. The $5700 \mathrm{~A}$ has an accuracy suitable to calibrate up to $8^{1 / 2}$ digit DMMs.

The instrument is equipped with a process of selfcalibration to compare its internal references of the different quantities then, in an automatic way, identifying anomalies in the value of the references themselves. This allows me to keep under control the instrument over time and after transport operations.

\section{ILC execution technical aspects}

The calibration was carried out with the traveling Standard in thermal equilibrium with the environment at a temperature of $23.0^{\circ} \mathrm{C}$, after a feeding period of at least $24 \mathrm{~h}$ with a sinusoidal voltage of $230 \mathrm{~V} \mathrm{rms}$, frequency $50.0 \mathrm{~Hz}$ and distortion less than $1 \%$. After performing successfully the SELF DIAG and CAL zero procedures on the calibrator, it was calibrated in the measuring points shown in Table 1.

Table 1. Measurement fields of the ILC regarding the calibration of a J. Fluke 5700 A MFC.

\begin{tabular}{|l|c|l|}
\hline \multicolumn{1}{|c|}{ Quantity } & Meas. range & Frequency range \\
\hline DC Voltage & $1 \mathrm{mV} \div 1000 \mathrm{~V}$ & \\
\hline AC Voltage & $1 \mathrm{mV} \div 1000 \mathrm{~V}$ & $40 \mathrm{~Hz} \div 1 \mathrm{MHz}$ \\
\hline DC Current & $10 \mu \mathrm{A} \div 10 \mathrm{~A}$ & \\
\hline AC Current & $100 \mu \mathrm{A} \div 10 \mathrm{~A}$ & $40 \mathrm{~Hz} \div 5 \mathrm{kHz}$ \\
\hline DC Resistance & $1 \Omega \div 100 \mathrm{M} \Omega$ & \\
\hline
\end{tabular}

\section{Analysis of the results}

To introduce this analysis it is helpful to clarify that the INRIM-Lab for multifunction instruments calibration and the accredited Laboratory under evaluation are both high level calibration Laboratories with traceability charts as shown in Fig. 2. For example the traceability chart of the INRIM-Lab for multifunction instruments calibration is shown in Fig. 4. 


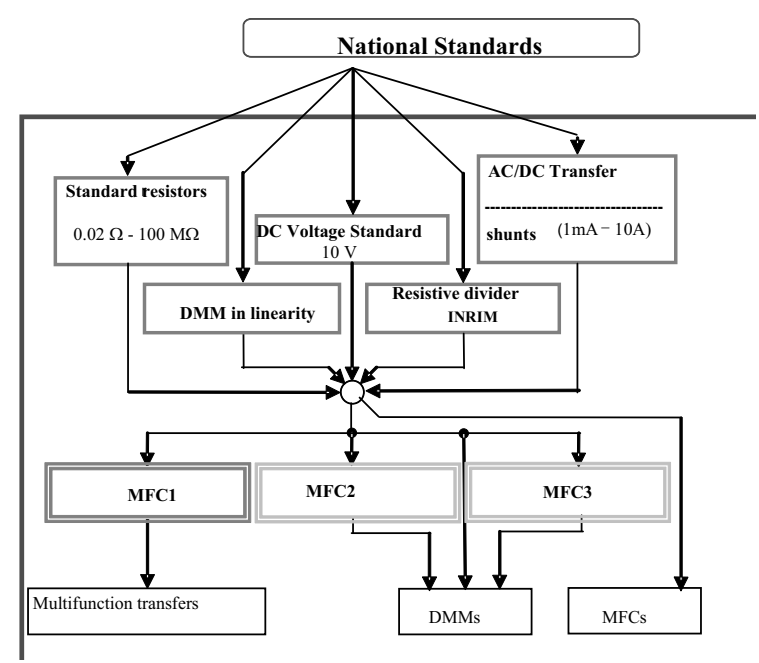

Figure 4. Traceability scheme of the INRIM-Lab for multifunction instruments calibration from INRIM National Standards.

The traceability chart of the accredited Laboratory participating at the ILC is very similar with some Standards of the same model in common with the INRIM-Lab and calibrated at INRIM. This situation implies to consider in the analysis of the results the effect of a partial correlation between INRIM-Lab and the Accredited Laboratory measurement uncertainties arising from the type B INRIM calibration uncertainties of the common Reference Standards of the two Laboratories both calibrated at INRIM vs. the National Standards. The traveling calibrator was calibrated twice by the Laboratory before and after its calibration at INRIM and was taken into account the Laboratory mean values to minimize the possible drift of the calibrator. For the evaluation of the comparison and of the Laboratory under accreditation renewal, the results for each measurement point were analysed considering as measurand a calibrator "relative error" defined as in the following (1) and (2). INRIM and Laboratory relative errors were evaluated respectively as:

$$
\begin{gathered}
E_{I}=\left(V_{I}-S_{I}\right) / S_{I} \\
E_{L}=\frac{\left(V_{L 1}-S_{L}\right)+\left(V_{L 2}-S_{L}\right)}{2 \times S_{L}}
\end{gathered}
$$

where $V_{I}$ and $S_{I}$ indicate respectively the quantity value provided by the calibrator and its setting shown on its display stated by the INRIM-Lab, $V_{L 1}$ and $V_{L 2}$ indicate the quantity values provided by the traveling calibrator stated by the Laboratory before and after its calibration at INRIM at the corresponding setting $I_{L}$.

It was defined for each measurement point a new measurand consisting in the difference between $E_{L}$ and $E_{I}$

$$
d=E_{L}-E_{I}
$$

whose relative standard uncertainty is:

$$
u^{2}(d)=\left[u^{2}\left(E_{I}\right)+u^{2}\left(E_{L}\right)-2 u_{E L} u_{E I} \times r\left(E_{L} E_{I}\right)\right]
$$

where $r\left(E_{L}, E_{I}\right)$ is the correlation factor between the uncertainties of the Laboratory and those of the INRIMLab, evaluated as follows:

- For DC Voltage: in each calibration point $r\left(E_{L}, E_{J}\right)$ was evaluated as the ratio between: the square of the type $B$ standard uncertainty of the calibration of the $10 \mathrm{~V}$ Reference Standard of both Laboratories (this uncertainty includes the uncertainties of the Josephson effect and that of the transfer to the $10 \mathrm{~V}$ reference Standards of the INRIM primary group) and the product of the two Laboratories declared standard uncertainties in the same measuring point;

- For AC Voltage: in each calibration point first of all, the quadratic sum of the DC Voltage type B standard uncertainty component (the same of DC Voltage) with the type B uncertainty of the DC Voltage values used to adjust the AC/DC transfer Standards of the two Laboratories was evaluated. Once established this value, $r\left(E_{L}, E_{I}\right)$ was obtained dividing it by the product of the two Laboratories declared standard uncertainties in the same measuring point;

- For DC Resistance in each calibration point $r\left(E_{L}, E_{I}\right)$ was evaluated as the ratio between the square of the type $B$ calibration standard uncertainty of the calibration of the Reference Standard Resistors at INRIM (that includes those of the Hall effect and of its transfer to the INRIM Resistance scale) and the product of the two Laboratories declared standard uncertainties in the same measuring point;

- For DC current in each calibration point first of all the quadratic sum of the DC Voltage type B standard uncertainty component with the type $\mathrm{B}$ calibration uncertainty of the resistors used to obtain the desired current values was evaluated. Once established this value, $r\left(E_{L}, E_{I}\right)$ was obtained dividing it by the product of the two Laboratories declared standard uncertainties in the same measuring point;

- For AC current $r\left(E_{L}, E_{I}\right)$ was evaluated in the same way of DC current as the common Standards AC/DC shunts are not calibrated, but only verified to respect their specifications. Finally, the normalized error En with respect to INRIM for each measurement point was evaluated as:

$$
E n=\frac{d}{U(d)}
$$

where $U(d)=2 u(d)$ at $2 \sigma$ level.

In Table 2 the results for AC Voltage are reported. In columns 8 and 9 are reported the type B standard calibration uncertainty of the $10 \mathrm{~V}$ Reference Standard of both Laboratories $u\left({ }_{D C V c o r r}\right)$ and the type $\mathrm{B}$ uncertainty of the DC Voltage values used to adjust the AC/DC transfer Standards $u\left(a_{a d j}\right)$ respectively while in column 10 the correlation coefficients for each measurement point are reported. 
Web of Conferences

Table 2. Analysis of the results of the ILC for AC Voltage.

\begin{tabular}{|c|c|c|c|c|c|c|c|c|c|c|c|}
\hline $\begin{array}{c}\text { Set } \\
\text { value } \\
(\mathrm{mV})\end{array}$ & $\begin{array}{c}\mathrm{f} \\
(\mathrm{kHz})\end{array}$ & $\begin{array}{c}E_{R} \\
\left(\times 10^{-6}\right)\end{array}$ & $\begin{array}{c}u\left(E_{R}\right) \\
\left(\times 10^{-6}\right)\end{array}$ & $\begin{array}{c}E_{L} \\
\left(\times 10^{-6}\right)\end{array}$ & $\begin{array}{c}u\left(E_{L}\right) \\
\left(\times 10^{-6}\right)\end{array}$ & $\begin{array}{c}d \\
\left(\times 10^{-6}\right)\end{array}$ & $\begin{array}{c}u(\text { DCVcorr }) \\
\left(\times 10^{-6}\right)\end{array}$ & $\begin{array}{l}u\left(a_{a d j}\right) \\
\left(\times 10^{-6}\right)\end{array}$ & $r$ & $\begin{array}{c}u(d) \\
\left(\times 10^{-6}\right)\end{array}$ & $E n$ \\
\hline 1.0 & 1 & 844 & 487 & 100.0 & 1300 & -744 & 0.1 & 96 & 0.0146 & 1381.6 & -0.27 \\
\hline 10.0 & 1 & 60 & 52 & -15.0 & 140 & -75 & 0.1 & 11.5 & 0.0182 & 148.5 & -0.25 \\
\hline \multirow{3}{*}{100} & 0.04 & 10 & 19 & 0.00 & 30 & -10 & 0.1 & 1 & 0.0021 & 35.5 & $\begin{array}{l}-0.14 \\
\end{array}$ \\
\hline & 1 & 1 & 19 & -10 & 30 & -11 & 0.1 & 1 & 0.0021 & 35.5 & -0.15 \\
\hline & 10 & -1 & 19 & -10 & 30 & -9 & 0.1 & 1 & 0.0021 & 35.5 & -0.12 \\
\hline 200 & 1 & 3 & 19 & 00 & 27.5 & -3 & 0.1 & 1 & 0.0024 & 33.1 & -0.04 \\
\hline \multicolumn{12}{|l|}{$(\mathrm{V})$} \\
\hline 0.3 & 1 & 17 & 18 & 1.67 & 23.3 & -15 & 0.1 & 0.9 & 0.0017 & 29.4 & -0.26 \\
\hline 0.5 & 1 & 5 & 15 & -50 & 22.0 & -10 & 0.1 & 0.9 & 0.0023 & 26.3 & -0.19 \\
\hline \multirow{5}{*}{1} & 0.04 & 14 & 14 & 40 & 22.0 & -10 & 0.1 & 0.6 & 0.0012 & 26.1 & -0.19 \\
\hline & 1 & 6 & 14 & -30 & 22.0 & -9 & 0.1 & 0.6 & 0.0012 & 26.1 & -0.17 \\
\hline & 100 & -43 & 20 & -35 & 55.0 & 8 & 0.1 & 0.6 & 0.0003 & 58.3 & 0.07 \\
\hline & 300 & -69 & 61 & 200 & 145.0 & 89 & 0.1 & 0.6 & 0.0000 & 157.1 & 0.28 \\
\hline & 1000 & -1207 & 178 & -550 & 750.0 & 657 & 0.1 & 0.6 & 0.0000 & 770.7 & 0.43 \\
\hline 2 & 1 & 5 & 14 & 1.75 & 22.5 & -4 & 0.1 & 0.6 & 0.0012 & 26.5 & -0.07 \\
\hline \multirow[t]{3}{*}{3} & 0.04 & 15 & 14 & 23.33 & 21.7 & 8 & 0.1 & 0.4 & 0.0004 & 25.8 & 0.16 \\
\hline & 1 & 13 & 14 & 150 & 21.7 & 2 & 0.1 & 0.4 & 0.0004 & 25.8 & 0.04 \\
\hline & 100 & 129 & 23 & 158.3 & 21.7 & 29 & 0.1 & 0.4 & 0.0003 & 31.6 & 0.46 \\
\hline 6 & 1 & -2 & 14 & 10.83 & 25.0 & 13 & 0.1 & 0.4 & 0.0004 & 28.4 & 0.23 \\
\hline \multirow{6}{*}{10} & 0.04 & 13 & 14 & 200 & 20.0 & 7 & 0.1 & 0.4 & 0.0006 & 24.1 & 0.14 \\
\hline & 1 & 5 & 14 & 150 & 20.0 & 10 & 0.1 & 0.4 & 0.0006 & 24.1 & 0.22 \\
\hline & 20 & 3 & 14 & 100 & 20.0 & 7 & 0.1 & 0.4 & 0.0006 & 24.1 & 0.15 \\
\hline & 100 & -47 & 23 & -10 & 50.0 & 37 & 0.1 & 0.4 & 0.0001 & 55.0 & 0.34 \\
\hline & 300 & -111 & 136 & -45 & 125.0 & 66 & 0.1 & 0.4 & 0.0000 & 184.7 & 0.18 \\
\hline & 1000 & -1368 & 324 & -850 & 750.0 & 518 & 0.1 & 0.4 & 0.0000 & 817.0 & 0.32 \\
\hline 20 & 1 & 5 & 14 & 200 & 20.0 & 15 & 0.1 & 0.4 & 0.0006 & 24.4 & 0.31 \\
\hline \multirow{3}{*}{30} & 0.04 & 21 & 18 & 300 & 21.7 & 9 & 0.1 & 0.7 & 0.0013 & 28.2 & 0.17 \\
\hline & 1 & 17 & 18 & 28.33 & 21.7 & 12 & 0.1 & 0.7 & 0.0013 & 28.2 & 0.21 \\
\hline & 100 & 120 & 50 & 156.7 & 58.3 & 37 & 0.1 & 0.7 & 0.0002 & 76.8 & 0.24 \\
\hline 60 & 1 & 8 & 18 & 200 & 20.8 & 12 & 0.1 & 0.7 & 0.0013 & 27.5 & 0.21 \\
\hline \multirow{4}{*}{100} & 0.04 & 25 & 18 & 300 & 21.0 & 5 & 0.1 & 0.8 & 0.0017 & 27.6 & 0.08 \\
\hline & 1 & 17 & 18 & 250 & 21.0 & 8 & 0.1 & 0.8 & 0.0017 & 27.6 & 0.14 \\
\hline & 20 & 7 & 18 & 200 & 21.0 & 13 & 0.1 & 0.8 & 0.0017 & 27.6 & 0.24 \\
\hline & 100 & -38 & 50 & -55 & 60.0 & -17 & 0.1 & 0.8 & 0.0002 & 78.1 & -0.11 \\
\hline 200 & 1 & 17 & 19 & 350 & 20.0 & 18 & 0.1 & 0.8 & 0.0018 & 27.2 & 0.33 \\
\hline \multirow{3}{*}{300} & 0.04 & 6 & 20 & 200 & 21.7 & 14 & 0.1 & 0.7 & 0.0010 & 29.5 & 0.24 \\
\hline & 1 & 11 & 20 & 200 & 21.7 & 9 & 0.1 & 0.7 & 0.0010 & 29.5 & 0.15 \\
\hline & 20 & -3 & 20 & 11.67 & 25.0 & 15 & 0.1 & 0.7 & 0.0009 & 32.0 & 0.23 \\
\hline 600 & 1 & 1 & 23 & 12.50 & 25.0 & 11 & 0.1 & 0.7 & 0.0008 & 33.6 & 0.17 \\
\hline \multirow{4}{*}{1000} & 0.04 & 3 & 29 & 100 & 25.0 & 7 & 0.1 & 0.7 & 0.0006 & 37.9 & 0.09 \\
\hline & 1 & 7 & 29 & 200 & 25.0 & 13 & 0.1 & 0.7 & 0.0006 & 37.9 & 0.17 \\
\hline & 20 & -19 & 45 & 00 & 80.0 & 19 & 0.1 & 0.7 & 0.0001 & 91.8 & 0.10 \\
\hline & 30 & -26 & 80 & -10 & 80.0 & 16 & 0.1 & 0.7 & 0.0001 & 113.1 & 0.07 \\
\hline
\end{tabular}

This work was made for all the measurement points of

Table 1. In Figures from 5 to 8 the $E n$ values for DC

Voltage, DC Resistance, DC Current and AC Current are reported. 


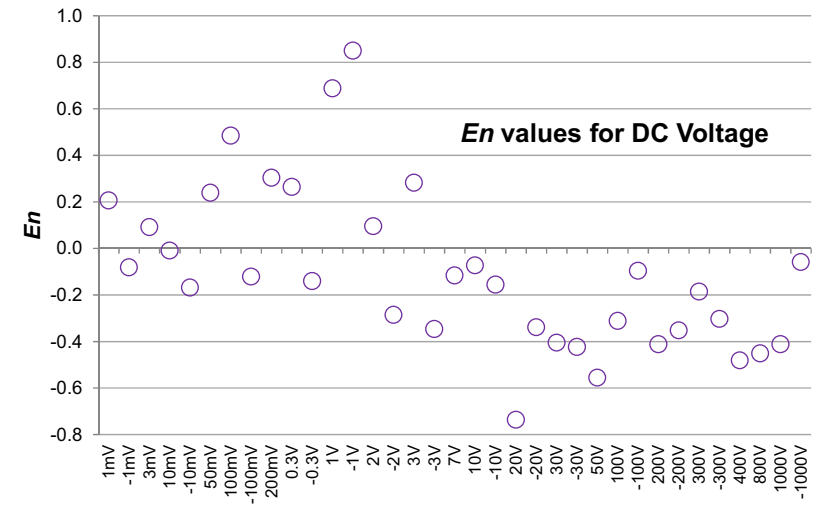

Figure 5. En values for DC Voltage.

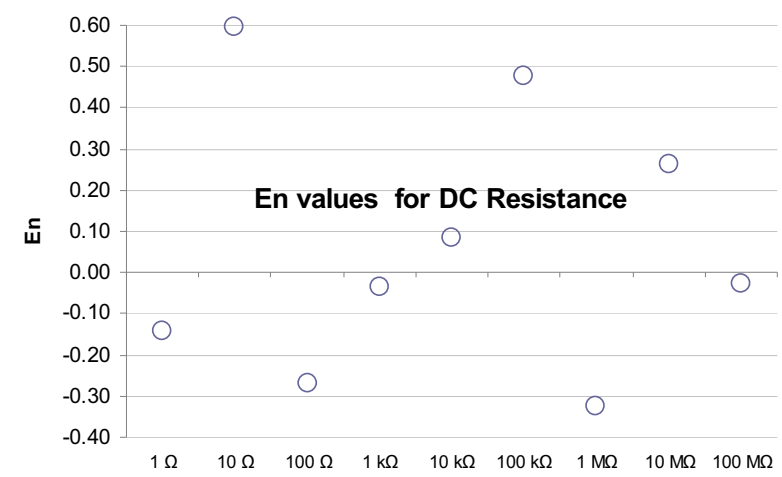

Figure 6. En values for DC Resistance.

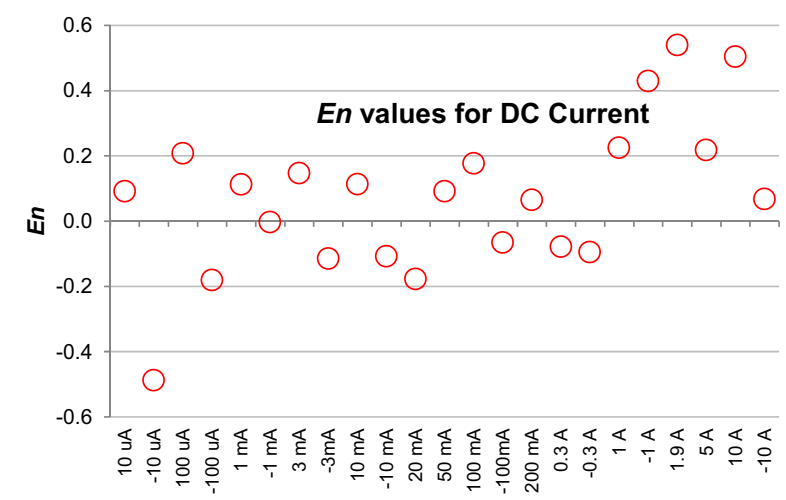

Figure 7. En values for DC Current.

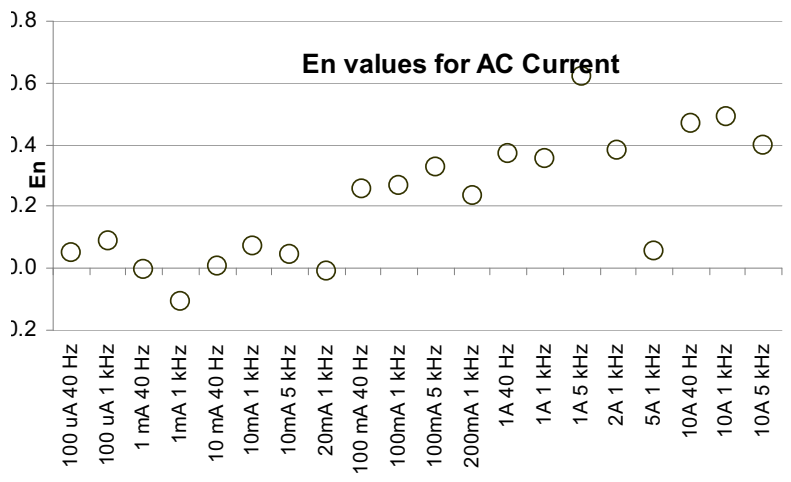

Figure 8. En values for AC Current.
From Table 2 and from Fig. 5 to 8 it can be seen that the $E n$ is always less than 1 for each measurement point of the comparison and in particular the mean absolute values were 0.3 for DC Voltages and DC Resistance and 0.2 for the other three electrical quantities. The only value to better analyse is the $-1 \mathrm{~V}$ of DC Voltage with a $E n$ of 0.8 . The definition of a criterion to individuate the correlation components between the two Laboratories can be very useful for future ILCs that INRIM will provide for these and similar kind of multifunction instruments. In addition, the used calibrator for the ILC seemed suitable to correctly evaluate the measurement capabilities of the high level calibration Laboratory under evaluation for its accreditation renewal.

\section{References}

1. Cassiago C., La Paglia G, Pogliano U., IEEE Trans. Meas., 49. 6, pp. 1206-1210, (2000).

2. Fluke Corporation, Calibration: Philosophy in Practice, Second Edition.

3. G. Rietveld, Artifact calibration: An evaluation of the Fluke 5700A series II calibrator, Rep. ISBN 909\0013\0322-4, (1999).

4. Capra P.P, Galliana F., arXiv:15054398v1 [physics.ins-det, (2015).

5. Oldham, N. ; Parker, M. Instrum. Meas. Tech. Conf., 1999. IMTC/99. Proceedings of the 16th IEEE Vol.: 3 DOI: 10.1109/IMTC.1999.776073 r: 1999 , Page(s): 1485-1487 (1999).

6. Cassiago, C.; Callegaro, L.; La Paglia, G. Instrumentation and Measurement Technology Conference, 2004. IMTC 04. Proceedings of the 21st IEEE Volume: 1 DOI: , Page(s): 102-105 Vol.1, (2004).

7. Galliana F., Capra P.P., Gasparotto E., Measurement . 45 , 3 , pp. 615-621, 2012).

8. Galliana F., Gasparotto E., Measurement, 52; p. 6470, (2014).

9. Hamilton C. A. IEEE Trans. Instr. Meas.. 54, 1, pp. 215-221, (2005).

10. Bosco GC., La Paglia G, Pogliano, U.and Zago G., in Proc. XIII IMEKO World Congr., Turin, Italy, pp.517-522, (1994).

11. Crisp P. B., MCSL Workshop and Symp., 1997, pp. 151-160, (1997).

12. JCGM 200, (2012).

\section{Discussion}

